\title{
ASYMPTOTIC INVERSION OF INCOMPLETE GAMMA FUNCTIONS
}

\author{
N. M. TEMME
}

\begin{abstract}
The normalized incomplete gamma functions $P(a, x)$ and $Q(a, x)$ are inverted for large values of the parameter $a$. That is, $x$-solutions of the equations

$$
P(a, x)=p, \quad Q(a, x)=q, \quad p \in[0,1], q=1-p,
$$

are considered, especially for large values of $a$. The approximations are obtained by using uniform asymptotic expansions of the incomplete gamma functions in which an error function is the dominant term. The inversion problem is started by inverting this error function term. Numerical results indicate that for obtaining an accuracy of four correct digits, the method can already be used for $a=2$, although $a$ is a large parameter. It is indicated that the method can be applied to other cumulative distribution functions.
\end{abstract}

\section{INTRODUCTION}

The incomplete gamma functions are defined by

$$
P(a, x)=\frac{1}{\Gamma(a)} \int_{0}^{x} t^{a-1} e^{-t} d t, \quad Q(a, x)=\frac{1}{\Gamma(a)} \int_{x}^{\infty} t^{a-1} e^{-t} d t,
$$

with

$$
P(a, x)+Q(a, x)=1 .
$$

We take $a>0$ and $x \geq 0$.

We consider the following inversion problem. Let $p \in[0,1]$ be given and $q=1-p$. Then we are interested in the $x$-value that solves the following two (equivalent) equations

$$
P(a, x)=p, \quad Q(a, x)=q,
$$

where $a$ is a fixed positive number. We are especially concerned with solving (1.3) for large values of $a$.

This problem is of importance in probability theory and mathematical statistics. Several approaches are available in the (statistical) literature, where often a first approximation of $x$ is constructed, based on asymptotic expansions, but this first approximation is not always reliable. Higher approximations may be

Received October 29, 1990; revised March 20, 1991.

1991 Mathematics Subject Classification. Primary 33B15, 33B20, 41A60, 65U05.

Key words and phrases. Incomplete gamma function, $\chi^{2}$ distribution, inversion of gamma distribution, asymptotic expansion. 
obtained by numerical inversion techniques, which require evaluation of the incomplete gamma functions. This may be rather time consuming, especially when $a$ is large.

In the present method we also use an asymptotic result. The approximation is quite accurate, especially when $a$ is large. It follows from numerical results, however, that a three-term asymptotic expansion already gives an accuracy of four significant digits for $a=2$, uniformly with respect to $p, q \in[0,1]$.

The method is rather general. In a final section we mention application of the same method on a wider class of cumulative distribution functions.

\section{UNIFORM ASYMPTOTIC EXPANSIONS OF $P$ AND $Q$}

The asymptotic inversion of equations (1.3) is based on the uniform asymptotic expansion of the incomplete gamma functions as given in [8]. First we summarize these results.

The incomplete gamma functions have the following representations:

$$
\begin{aligned}
& P(a, x)=\frac{1}{2} \operatorname{erfc}(-\eta \sqrt{a / 2})-R_{a}(\eta), \\
& Q(a, x)=\frac{1}{2} \operatorname{erfc}(\eta \sqrt{a / 2})+R_{a}(\eta)
\end{aligned}
$$

here, erfc is the error function defined by

$$
\operatorname{erfc} z=\frac{2}{\sqrt{\pi}} \int_{z}^{\infty} e^{-t^{2}} d t
$$

The real parameter $\eta$ in (2.1) is defined by

$$
\frac{1}{2} \eta^{2}=\lambda-1-\ln \lambda, \quad \lambda=x / a, \operatorname{sign}(\eta)=\operatorname{sign}(\lambda-1) .
$$

For the function $R_{a}(\eta)$ we derived an asymptotic expansion. Writing

$$
R_{a}(\eta)=\frac{e^{-a \eta^{2} / 2}}{\sqrt{2 \pi a}} S_{a}(\eta)
$$

we have

$$
S_{a}(\eta) \sim \sum_{n=0}^{\infty} \frac{C_{n}(\eta)}{a^{n}} \quad \text { as } a \rightarrow \infty, \eta \in \mathbb{R} .
$$

No restrictions on $\eta$ are needed. In fact, (2.5) holds uniformly with respect to $\eta \in \mathbb{R}$ (and in a larger domain of the complex plane). In other words, (2.5) holds uniformly with respect to $\lambda \in[0, \infty)$ or with respect to $x \in[0, \infty)$. In particular, the expansion is valid in a neighborhood of $\lambda=1 \quad(x=a)$, a turning point in the behavior of the incomplete gamma functions for large values of the parameter $a$. The first two coefficients in (2.5) are

$$
\begin{aligned}
& C_{0}(\eta)=\frac{1}{\lambda-1}-\frac{1}{\eta}, \\
& C_{1}(\eta)=\frac{1}{\eta^{3}}-\frac{1}{(\lambda-1)^{3}}-\frac{1}{(\lambda-1)^{2}}-\frac{1}{12(\lambda-1)} .
\end{aligned}
$$

These two (and all higher coefficients) have a removable singularity at $\eta=0$ $(\lambda=1, x=a)$. All $C_{n}(\eta)$ are analytic at the origin. The higher coefficients can be obtained from the recursion

$$
\eta C_{n}(\eta)=\frac{d}{d \eta} C_{n-1}(\eta)+\frac{\eta}{\lambda-1} \gamma_{n}, \quad n \geq 1
$$


where the numbers $\gamma_{n}$ appear in the well-known asymptotic expansion of the Euler gamma function. That is,

$$
\Gamma^{*}(a) \sim \sum_{n=0}^{\infty}(-1)^{n} \gamma_{n} a^{-n}, \quad \frac{1}{\Gamma^{*}(a)} \sim \sum_{n=0}^{\infty} \gamma_{n} a^{-n}, \quad a \rightarrow \infty,
$$

where

$$
\Gamma^{*}(a)=\sqrt{\frac{a}{2 \pi}} e^{a} a^{-a} \Gamma(a), \quad a>0 .
$$

The first few $\gamma_{n}$ are

$$
\gamma_{0}=1, \quad \gamma_{1}=-\frac{1}{12}, \quad \gamma_{2}=\frac{1}{288}, \quad \gamma_{3}=\frac{139}{51840} .
$$

\section{ASYMPTOTIC INVERSION OF THE INCOMPLETE GAMMA FUNCTIONS}

We perform the inversion of the equations (1.3) with respect to the parameter $\eta$, by using representations (2.1) with large values of $a$. Afterwards, we have to compute $\lambda$ and $x$ from (2.3). We concentrate on the second equation in (1.3). Let us rewrite the inversion problem in the form

$$
\frac{1}{2} \operatorname{erfc}(\eta \sqrt{a / 2})+R_{a}(\eta)=q, \quad q \in[0,1],
$$

which is equivalent to the second equation in (1.3), and we denote the solution of the above equation by $\eta(q, a)$.

To start the procedure, we consider $R_{a}(\eta)$ in (3.1) as a perturbation, and we define the number $\eta_{0}=\eta_{0}(q, a)$ as the real number that satisfies the equation

$$
\frac{1}{2} \operatorname{erfc}\left(\eta_{0} \sqrt{a / 2}\right)=q \text {. }
$$

Known values are

$$
\eta_{0}(0, a)=+\infty, \quad \eta_{0}\left(\frac{1}{2}, a\right)=0, \quad \eta_{0}(1, a)=-\infty
$$

Note the symmetry $\eta_{0}(q, a)=-\eta_{0}(p, a)$. Computation of $\eta_{0}$ requires an inversion of the error function, but this problem has been satisfactorily solved in the literature (see $[3,7])$.

The value $\eta$ defined by (3.1) is, for large values of $a$, approximated by the value $\eta_{0}$. We write

$$
\eta(q, a)=\eta_{0}(q, a)+\varepsilon\left(\eta_{0}, a\right),
$$

and we try to determine the function $\varepsilon$. It appears that we can expand this quantity in the form

$$
\varepsilon\left(\eta_{0}, a\right) \sim \frac{\varepsilon_{1}}{a}+\frac{\varepsilon_{2}}{a^{2}}+\frac{\varepsilon_{3}}{a^{3}}+\cdots,
$$

as $a \rightarrow \infty$. The coefficients $\varepsilon_{i}$ can be written explicitly as functions of $\eta_{0}$.

We first remark that (3.1) yields the relation

$$
\frac{d q}{d \eta}=\frac{d}{d \eta} Q(a, x)=\frac{d}{d x} Q(a, x) \frac{d x}{d \eta} .
$$

Using (1.1) and (2.3), we obtain after straightforward calculations

$$
\frac{d q}{d \eta}=-\frac{1}{\Gamma^{*}(a)} \sqrt{\frac{a}{2 \pi}} f(\eta) e^{-a \eta^{2} / 2},
$$


where $\Gamma^{*}(a)$ is defined in (2.9), and

$$
f(\eta)=\frac{\eta}{\lambda-1}
$$

the relation between $\eta$ and $\lambda$ being given in (2.3). For small values of $\eta$ we can expand

$$
f(\eta)=1-\frac{1}{3} \eta+\frac{1}{12} \eta^{2}+\cdots
$$

From (3.2) we obtain

$$
\frac{d q}{d \eta_{0}}=-\sqrt{\frac{a}{2 \pi}} e^{-a \eta_{0}^{2} / 2} .
$$

Upon dividing these two differential equations, we eliminate $q$, although it is still present in $\eta_{0}$. We thus obtain

$$
\frac{d \eta}{d \eta_{0}}=\frac{\Gamma^{*}(a)}{f(\eta)} e^{a\left(\eta^{2}-\eta_{0}^{2}\right) / 2}, \quad-\infty<\eta_{0}<\infty .
$$

Substitution of (3.3) gives the differential equation

$$
f\left(\eta_{0}+\varepsilon\right)\left[1+\frac{d \varepsilon}{d \eta_{0}}\right]=\Gamma^{*}(a) e^{a \varepsilon\left(\eta_{0}+\varepsilon / 2\right)},
$$

a relation between $\varepsilon$ and $\eta_{0}$, with $a$ a (large) parameter.

It is convenient to write $\eta$ in place of $\eta_{0}$. That is, we try to find the function $\varepsilon=\varepsilon(\eta, a)$ that satisfies the equation

$$
f(\eta+\varepsilon)\left[1+\frac{d \varepsilon}{d \eta}\right]=\Gamma^{*}(a) e^{a \varepsilon(\eta+\varepsilon / 2)} .
$$

When we have obtained the solution $\varepsilon(\eta, a)$ (or an approximation), we write it as $\varepsilon\left(\eta_{0}, a\right)$ and the final value of $\eta$ follows from (3.3). The parameters $\lambda$ and $x$ of the incomplete gamma function then follow from inversion of the first relation in $(2.3)$.

\section{Determination OF THE COEFFICIENTS $\varepsilon_{i}$}

For large values of $a$ we have $\Gamma^{*}(a)=1+\mathscr{O}\left(a^{-1}\right)$ (see (2.8)). Comparing dominant terms in (3.10), we infer that the first coefficient $\varepsilon_{1}$ in (3.4) is defined by $f(\eta)=e^{\eta \varepsilon_{1}}$, giving

$$
\varepsilon_{1}=\frac{1}{\eta} \ln f(\eta)
$$

It is not difficult to verify that $f$ is positive on $\mathbb{R}, f(0)=1$, and that $f$ is analytic in a neighborhood of $\eta=0$. It follows that $\varepsilon_{1}=\varepsilon_{1}(\eta)$ is an analytic function on $\mathbb{R}$. For small values of $\eta$ we have, using (3.7),

$$
\varepsilon_{1}=-\frac{1}{3}+\frac{1}{36} \eta+\frac{1}{1620} \eta^{2}+\cdots
$$

The function $\varepsilon_{1}(\eta)$ is nonvanishing on $\mathbb{R}$ (and hence negative). To show this, consider the equation $f^{2}(\eta)=1$. From (3.6) and the first relation in (2.3) it follows that the corresponding $\lambda$-value should satisfy

$$
-\ln \lambda=(\lambda-1)(2 \lambda-3) \text {. }
$$


This equation has only one real solution $\lambda=1$, which gives $\eta=0$. However, for this value, $\varepsilon_{1}$ equals $-\frac{1}{3}$.

Further coefficients in (3.4) are obtained by using standard perturbation methods. We need the expansion of $\Gamma^{*}(a)$ given in (2.8), and

$$
f(\eta+\varepsilon)=f(\eta)+\varepsilon f^{\prime}(\eta)+\frac{1}{2} \varepsilon^{2} f^{\prime \prime}(\eta)+\cdots,
$$

in which (3.4) is substituted to obtain an expansion in powers of $a^{-1}$. Putting all this in (3.10), we find by comparing terms with equal powers of $a^{-1}$ :

$$
\begin{aligned}
& \varepsilon_{2}=\frac{1}{12 \eta f}\left(12 f \varepsilon_{1}^{\prime}+12 f^{\prime} \varepsilon_{1}-f-6 f \varepsilon_{1}^{2}\right), \\
& \varepsilon_{3}=\frac{1}{288 \eta f}\left(288 f \varepsilon_{2}^{\prime}+288 f^{\prime} \varepsilon_{1} \varepsilon_{1}^{\prime}-24 f \varepsilon_{1}^{\prime}+288 f^{\prime} \varepsilon_{2}+144 f^{\prime \prime} \varepsilon_{1}^{2}-24 f^{\prime} \varepsilon_{1}\right. \\
& \left.+f-288 f \varepsilon_{1} \varepsilon_{2}-144 f \varepsilon_{2}^{2} \eta^{2}-144 f \varepsilon_{2} \eta \varepsilon_{1}^{2}-36 f \varepsilon_{1}^{4}\right), \\
& \varepsilon_{4}=\frac{1}{51840 \eta f}\left(51840 f^{\prime \prime} \varepsilon_{1} \varepsilon_{2}-4320 f \varepsilon_{2}^{\prime}+180 f \varepsilon_{1}^{\prime}+180 f^{\prime} \varepsilon_{1}-4320 f^{\prime} \varepsilon_{2}\right. \\
& +51840 f^{\prime} \varepsilon_{3}+51840 f \varepsilon_{3}^{\prime}+139 f-2160 f^{\prime \prime} \varepsilon_{1}^{2}+8640 f^{(3)} \varepsilon_{1}^{3} \\
& -25920 f \varepsilon_{2}^{2}-1080 f \varepsilon_{1}^{6}-4320 f^{\prime} \varepsilon_{1} \varepsilon_{1}^{\prime}-51840 f \varepsilon_{1} \varepsilon_{3} \\
& -25920 f \varepsilon_{1}^{3} \varepsilon_{2}-8640 f \varepsilon_{2}^{3} \eta^{3}+51840 f^{\prime} \varepsilon_{2} \varepsilon_{1}^{\prime}+25920 f^{\prime \prime} \varepsilon_{1}^{2} \varepsilon_{1}^{\prime} \\
& -51840 f \varepsilon_{2}^{2} \eta \varepsilon_{1}-51840 \varepsilon_{2} \eta^{2} \varepsilon_{3}-25920 f \varepsilon_{1}^{2} \varepsilon_{3} \eta \\
& \left.-12960 f \varepsilon_{2}^{2} \eta^{2} \varepsilon_{1}^{2}-6480 f \varepsilon_{2} \eta \varepsilon_{1}^{4}+51840 f^{\prime} \varepsilon_{1} \varepsilon_{2}^{\prime}\right) .
\end{aligned}
$$

The derivatives $f^{\prime}, \varepsilon_{1}^{\prime}$, etc., are with respect to $\eta$, and evaluated at $\eta$. It will be understood that the complexity for obtaining higher-order terms is considerable. The terms shown so far have been obtained by symbolic manipulation.

\section{EXPANSIONS OF THE COEFFICIENTS $\varepsilon_{i}$}

The singularities of the mapping $\lambda \rightarrow \eta$, the first relation in (2.3), follow from the zeros or poles of $d \eta / d \lambda=(\lambda-1) /(\lambda \eta) ; \lambda=0$ is mapped to infinity. A second candidate is $\lambda=1$ with corresponding point $\eta=0$, a regular point. However, when $\lambda=\exp (2 \pi i n)$, with $n= \pm 1, \pm 2, \ldots$, the quantity $d \eta / d \lambda$ vanishes. Corresponding $\eta$-values satisfying $\frac{1}{2} \eta_{n}^{2}=-2 \pi i n$ are singular points of the mapping, and singular points of the function $f$ defined in (3.6). For $n= \pm 1$ we obtain $2 \sqrt{\pi} \exp \left( \pm \frac{3}{4} \pi i\right)$.

It follows that $f$ is analytic in a strip $|\Im \eta|<\sqrt{2 \pi}$, and that it can be expanded in a Taylor series around the origin with radius of convergence $2 \sqrt{\pi}$. All $\varepsilon_{i}$ have similar analytic properties. That is, the coefficients $\varepsilon_{i}$ can be expanded in series

$$
\varepsilon_{i}=\sum_{n=0}^{\infty} c_{i, n} \eta^{n}, \quad|\eta|<2 \sqrt{\pi}, i=1,2,3, \ldots
$$

The representations of $\varepsilon_{i}$ given in the previous section are not suitable for numerical computations. First, because of the appearance of derivatives of $f$ and 
$\varepsilon_{i}$, secondly, because of the complexity of the expressions. To facilitate numerical evaluations of $\varepsilon_{1}, \ldots, \varepsilon_{4}$, we provide the following Taylor expansions:

$$
\begin{aligned}
\varepsilon_{1}= & -\frac{1}{3}+\frac{1}{36} \eta+\frac{1}{1620} \eta^{2}-\frac{7}{6480} \eta^{2}+\frac{5}{18144} \eta^{4}-\frac{11}{382725} \eta^{5}-\frac{101}{16329600} \eta^{6} \\
& +\frac{37}{9797760} \eta^{7}-\frac{454973}{498845952000} \eta^{8}+\frac{1231}{15913705500} \eta^{9}+\frac{2745493}{84737299046400} \eta^{10} \\
& -\frac{2152217}{127673385840000} \eta^{11}+\frac{119937661}{30505427656704000} \eta^{12} \\
& -\frac{449}{1595917323000} \eta^{13}-\frac{756882301459}{4455179048226816000000} \eta^{14} \\
& +\frac{1269400547}{153146779782796800000} \eta^{15}-\frac{3224618478943}{170264214140233973760000} \eta^{16}+\cdots, \\
\varepsilon_{2}= & -\frac{7}{405}-\frac{7}{2592} \eta+\frac{533}{204120} \eta^{2}-\frac{1579}{2099520} \eta^{3}+\frac{109}{1749600} \eta^{4}+\frac{10217}{251942400} \eta^{5} \\
& -\frac{9281803}{436490208000} \eta^{6}+\frac{919081}{185177664000} \eta^{7}-\frac{100824673}{571976768563200} \eta^{8} \\
& -\frac{311266223}{899963447040000} \eta^{9}+\frac{52310527831}{343186061137920000} \eta^{10}+\cdots \\
\varepsilon_{3}= & +\frac{449}{102060}-\frac{63149}{20995200} \eta+\frac{29233}{36741600} \eta^{2}+\frac{346793}{5290790400} \eta^{3} \\
& -\frac{18442139}{130947062400} \eta^{4}+\frac{14408797}{246903552000} \eta^{5}-\frac{1359578327}{129994720128000} \eta^{6} \\
& -\frac{69980826653}{39598391669760000} \eta^{7}+\frac{987512909021}{514779091706880000} \eta^{8}+\cdots, \\
\varepsilon_{4}= & +\frac{319}{183708}-\frac{269383}{4232632320} \eta-\frac{449882243}{982102968000} \eta^{2}+\frac{1981235233}{6666395904000} \eta^{3} \\
& -\frac{16968489929}{194992080192000} \eta^{4}-\frac{16004851139}{26398927779840000} \eta^{5}+\frac{636178018081}{48260539847520000} \eta^{6}+\cdots
\end{aligned}
$$

On the other hand, for larger values of $\eta$ we need representations free of derivatives. The derivatives of $f$ can be eliminated by using

$$
\begin{aligned}
& f^{\prime}=-f\left(-1+f^{2}+f \eta\right) / \eta \\
& f^{\prime \prime}=+f^{2}\left(-3 \eta-3 f+3 f^{3}+5 f^{2} \eta+2 \eta^{2} f\right) / \eta^{2} \\
& f^{(3)}=-f^{2}\left(-30 f^{2} \eta-12 \eta^{2} f-18 f^{3}+15 f^{5}+3 f+35 f^{4} \eta+26 f^{3} \eta^{2}\right.\left.+6 f^{2} \eta^{3}+3 \eta\right) / \eta^{3} \\
& f^{(4)}=+f^{3}\left(-350 f^{3} \eta-260 f^{2} \eta^{2}-60 f \eta^{3}-150 f^{4}+75 f \eta+30 \eta^{2}+45 f^{2}\right. \\
& \\
&\left.\quad+105 f^{6}+154 f^{3} \eta^{3}+340 f^{4} \eta^{2}+24 f^{2} \eta^{4}+315 f^{5} \eta\right) / \eta^{4} .
\end{aligned}
$$

The first relation easily follows from (3.6) and the first relation in (2.3). Using these relations in the earlier expressions for $\varepsilon_{i}$, and eliminating the derivatives of previous $\varepsilon_{i}$, it follows that we can write $\eta^{2 i-1} \varepsilon_{i}$ as a polynomial in $\eta, f, \varepsilon_{1}$ :

$$
\begin{aligned}
12 \eta^{3} \varepsilon_{2}= & +12-12 f^{2}-12 f \eta-12 f^{2} \eta \varepsilon_{1}-12 f \eta^{2} \varepsilon_{1}-\eta^{2}-6 \eta^{2} \varepsilon_{1}^{2} \\
12 \eta^{5} \varepsilon_{3}= & -30+12 f^{2} \eta \varepsilon_{1}+12 f \eta^{2} \varepsilon_{1}+24 f^{2} \eta^{3} \varepsilon_{1}+6 \varepsilon_{1}^{3} \eta^{3}-12 f^{2}+60 f^{3} \eta^{2} \varepsilon_{1} \\
& +31 f^{2} \eta^{2}+72 f^{3} \eta+42 f^{4}+18 f^{3} \eta^{3} \varepsilon_{1}^{2}+6 f^{2} \eta^{4} \varepsilon_{1}^{2}+36 f^{4} \eta \varepsilon_{1} \\
& +12 \varepsilon_{1}^{2} \eta^{3} f+12 \varepsilon_{1}^{2} \eta^{2} f^{2}-12 \eta \varepsilon_{1}+\eta^{3} \varepsilon_{1}+f \eta^{3}-12 f \eta+12 \varepsilon_{1}^{2} \eta^{2} f^{4}
\end{aligned}
$$




$$
\begin{aligned}
1440 \eta^{7} \varepsilon_{4}= & +2880 f^{2} \eta \varepsilon_{1}+2880 f \eta^{2} \varepsilon_{1}-3840 f^{2} \eta^{3} \varepsilon_{1}+5040 f^{2}-7200 f^{3} \eta^{2} \varepsilon_{1} \\
& -480 f^{3} \varepsilon_{1}^{3} \eta^{6}-1920 f^{6} \varepsilon_{1}^{3} \eta^{3}-1440 f^{4} \varepsilon_{1}^{3} \eta^{3}-4080 f^{5} \eta^{4} \varepsilon_{1}^{3}+17040 \\
& +2160 \eta^{2} \varepsilon_{1}^{2}+2040 f^{2} \eta^{2}+5760 f^{3} \eta-61200 f^{5} \eta-47160 f^{4} \eta^{2} \\
& -11880 f^{3} \eta^{3}+3600 f^{4}-2640 f^{4} \varepsilon_{1}^{3} \eta^{5}-8640 f^{3} \eta^{3} \varepsilon_{1}^{2} \\
& -3600 f^{2} \eta^{4} \varepsilon_{1}^{2}-3600 f^{4} \eta \varepsilon_{1}-24480 f^{6} \eta \varepsilon_{1}-2160 \varepsilon_{1}^{2} \eta^{3} f \\
& -2160 \varepsilon_{1}^{2} \eta^{2} f^{2}+4 \eta^{6}+3600 \eta \varepsilon_{1}+120 \eta^{2}-5 \eta^{4}-25680 f^{6} \\
& -240 f^{2} \eta^{4}-120 f \eta^{3}+5040 f \eta-2160 \varepsilon_{1}^{3} \eta^{3} f^{2}-2160 \varepsilon_{1}^{3} \eta^{4} f \\
& -2160 f^{3} \eta^{4} \varepsilon_{1}^{3}-720 f^{2} \eta^{5} \varepsilon_{1}^{3}-120 f^{2} \eta^{5} \varepsilon_{1}-10080 f^{6} \eta^{2} \varepsilon_{1}^{2} \\
& -900 \varepsilon_{1}^{4} \eta^{4}-23040 f^{5} \eta^{3} \varepsilon_{1}^{2}-16560 f^{4} \eta^{4} \varepsilon_{1}^{2}-3600 f^{3} \eta^{5} \varepsilon_{1}^{2} \\
& -240 \eta^{4} \varepsilon_{1} f-5040 \varepsilon_{1}^{2} \eta^{2} f^{4}-57600 f^{5} \eta^{2} \varepsilon_{1}-43440 f^{4} \eta^{3} \varepsilon_{1} \\
& -10440 f^{3} \eta^{4} \varepsilon_{1}-180 \eta^{4} \varepsilon_{1}^{2} .
\end{aligned}
$$

The coefficients $\varepsilon_{1}, \ldots, \varepsilon_{4}$ are bounded on $\mathbb{R}$. To show this, one needs

$$
f(\eta) \sim-\eta, \quad \eta \rightarrow-\infty, \quad f(\eta) \sim 2 \eta^{-1}, \quad \eta \rightarrow+\infty
$$

and the above representations of $\varepsilon_{i}$. We find

$$
\varepsilon_{1} \sim \mp \frac{\ln |\eta|}{\eta}, \quad \varepsilon_{2} \sim-\frac{1}{12 \eta}, \quad \varepsilon_{3} \sim \frac{\varepsilon_{1}}{12 \eta^{2}}, \quad \varepsilon_{4} \sim \frac{1}{360 \eta}
$$

as $\eta \rightarrow \pm \infty$. In deriving the behavior at $-\infty$, one should take into account that (see (3.6) and the first relation in (2.3))

$$
f(\eta)+\eta=\frac{\lambda \eta}{\lambda-1} \sim-\eta e^{-\eta^{2} / 2}, \quad \eta \rightarrow-\infty
$$

\section{NUMERICAL EXAMPLES}

In a separate publication we present numerical approximations (in the form of rational functions) for the coefficients $\varepsilon_{i}$, together with a computer program for computing the inverse of the error function and the incomplete gamma functions. The inversion of the first relation in (2.3), that is, the computation of $\lambda$ when $\eta$ is given, will be considered as well. In this section we present some first numerical results, which show the power of the asymptotic method.

When $p=q=\frac{1}{2}$, the asymptotics is quite simple. Then $\eta_{0}$ of (3.2) equals zero, and from (4.2) and the expansions in $\S 5$ we obtain (3.3)-(3.4) in the form

$$
\eta \sim-\frac{1}{3} a^{-1}-\frac{7}{405} a^{-2}+\frac{449}{102060} a^{-3}+\frac{319}{183708} a^{-4}+\cdots .
$$

In this case we give an expansion of the requested value $x$. Recall that $x=a \lambda$ (see (2.3)), and that $\lambda$ can be obtained from the first relation in (2.3) with $\eta$ given by (6.1). Inverting

$$
\frac{1}{2} \eta^{2}=\frac{1}{2}(\lambda-1)^{2}-\frac{1}{3}(\lambda-1)^{3}+\frac{1}{4}(\lambda-1)^{4}+\cdots,
$$


we obtain

$$
\lambda=1+\eta+\frac{1}{3} \eta^{2}+\frac{1}{36} \eta^{3}-\frac{1}{270} \eta^{4}+\frac{1}{4320} \eta^{5}+\cdots .
$$

Substituting (6.1), we have

$$
x \sim a\left(1-\frac{1}{3} a^{-1}+\frac{8}{405} a^{-2}+\frac{184}{25515} a^{-3}+\frac{2248}{3444525} a^{-4}+\cdots\right) .
$$

When $a=1$ and $q=\frac{1}{2}$, the equations in (1.3) reduce to $e^{-x}=\frac{1}{2}$, with solution $x=\ln 2=0.693147 \ldots$, while expansion (6.2) gives $x \sim 0.694 \ldots$, an accuracy of about three digits. When $a=2$ and $q=\frac{1}{2}$, the equations in (1.3) become $(1+x) e^{-x}=\frac{1}{2}$, with solution $x=1.6783469 \ldots$; in this case our expansion (6.2) gives $x \sim 1.67842 \ldots$, an accuracy of four significant digits. This shows that (6.2) is quite accurate for small values of the (large) parameter $a$. Computer experiments show that for other $q$-values the results are of the same kind (see Table 6.1).

In a second example we take $a=2$ and $q=0.1$; inverting (3.2), we obtain $\eta_{0}=0.9061938$. Using (3.4), we compute

$$
\eta \sim \eta_{0}-0.308292 / 2-0.0180893 / 4+0.0023105 / 8=0.747814 \text {. }
$$

An inversion of the first relation in (2.3) gives $\lambda=1.944743$, and hence $x=$ $2 \lambda=3.889486$. Computing $Q(2, x)$ with this value of $x$ gives 0.1000186 , an accuracy of four digits. A more accurate value of $x$ can be obtained by a Newton-Raphson method, giving $x=3.8897202$. It follows that the value of $x$ obtained by the asymptotic method is accurate within four significant digits.

In Table 6.1 we give more results of numerical experiments. We have used (3.4) with three terms. The first column under each $a$-value gives the relative accuracy $\left|x_{a}-x\right| / x$, where $x_{a}$ is the result of the asymptotic method, and $x$ is a more accurate value obtained by a Newton-Raphson method. The second column under each $a$-value gives the relative errors $\left|Q\left(a, x_{a}\right)-q\right| / q$.

TABLE 6.1

Relative errors $\left|x_{a}-x\right| / x$ and $\left|Q\left(a, x_{a}\right)-q\right| / q$ for several values of $q$ and $a ; x_{a}$ is obtained from the asymptotic expansion (3.4), $x$ is a more accurate value.

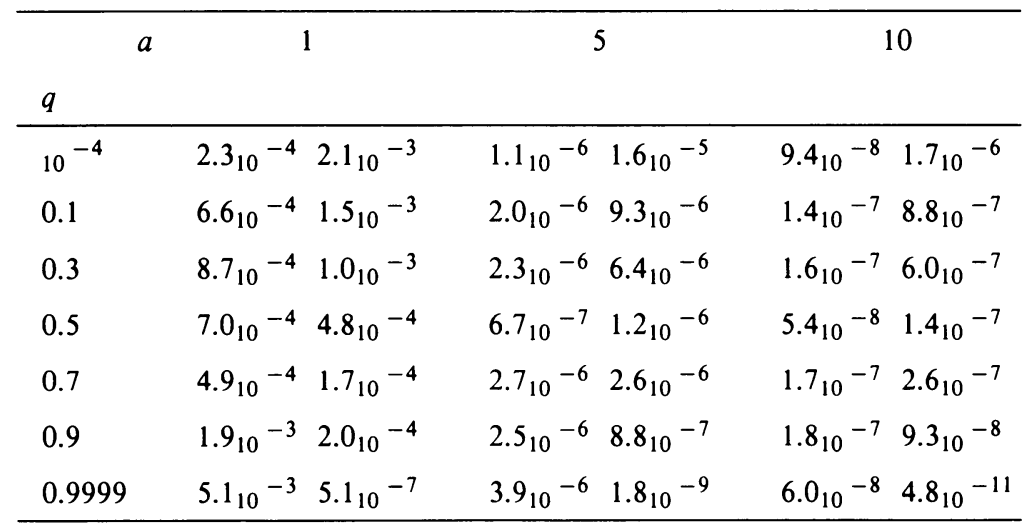


The numerical inversion of the error function, equation (3.2), can be based on formula 26.2 .23 of [1], where the inversion is written in terms of the function $Q(x)=\frac{1}{2} \operatorname{erfc}(x / \sqrt{2})$. The equation $Q\left(x_{p}\right)=p$ is considered with $0<p \leq$ 0.5 , but symmetry of the $Q$-function can be used for $0.5<p<1$. In $[3,7]$ more results can be found on the inverse of the error function.

Inversion of the incomplete gamma functions is considered in [1] in terms of the chi-square distribution (see formulas 26.4.16-18). In [2] an algorithm is published (in Fortran). In [4] several algorithms are discussed. In [5] an algorithm in Fortran is given for the incomplete gamma functions and their inverses. In [6] asymptotic methods are used; in our notation: (1.3) is considered for small values of $q$, with $a$ fixed. These results can be used in addition to our results when $q$ is small and $a \in(0,2)$, say.

\section{Generalizations}

The method described in the previous sections can be applied to other cumulative distribution functions. Consider the function

$$
F_{a}(\eta)=\sqrt{\frac{a}{2 \pi}} \int_{-\infty}^{\eta} e^{-a \zeta^{2} / 2} f(\zeta) d \zeta
$$

where $a>0$ and $\eta \in \mathbb{R}$. We assume that $f$ is an analytic function in a domain containing the real axis, and that $f$ is positive on $\mathbb{R}$ with the normalization $f(0)=1$. In [9] it is shown that several well-known distribution functions can be written in this form, including the incomplete gamma and beta functions. It is also shown that the representation

$$
F_{a}(\eta)=\frac{1}{2} \operatorname{erfc}(-\eta \sqrt{a / 2}) F_{a}(\infty)+R_{a}(\eta)
$$

holds, where $R_{a}(\eta)$ can be expanded as in (2.4)-(2.5). $F_{a}(\infty)$ is the complete integral, and can be expanded in the form

$$
F_{a}(\infty) \sim \sum_{n=0}^{\infty} \frac{A_{n}}{a^{n}} \quad \text { as } a \rightarrow \infty, A_{0}=1
$$

By dividing both sides of $(7.1)$ by $F_{a}(\infty)$, we obtain a further normalization, which is typical for distribution functions.

The inversion of the equation $F_{a}(\eta) / F_{a}(\infty)=q$, with $q \in[0,1]$ and $a$ a given (large) number, can be performed as in the case of the incomplete gamma functions. As in (3.2), let $\eta_{0}$ be the real number satisfying the equation

$$
\frac{1}{2} \operatorname{erfc}\left(-\eta_{0} \sqrt{a / 2}\right)=q
$$

Then the desired value $\eta$ is written as in (3.3), and an expansion like (3.4) can be obtained by deriving the differential equation (3.8), with $f$ of (7.1) and $\Gamma^{*}(a)$ replaced with $F_{a}(\infty)$.

From [9] it follows that the incomplete beta function defined as

$$
I_{x}(p, q)=\frac{1}{B(p, q)} \int_{0}^{x} t^{p-1}(1-t)^{q-1} d t, \quad x \in[0,1], p>0, q>0,
$$

with $B(p, q)=\Gamma(p) \Gamma(q) / \Gamma(p+q)$, can be inverted in this way. The large parameter is $a=p+q$, and the inversion method described above holds for the case that both $p$ and $q$ are large. That is, a representation as in (7.1) and 
(7.2) can be given when the beta density is not too skew. The condition on $p$ and $q$ is: when we write

$$
p=a \sin ^{2} \theta, \quad q=a \cos ^{2} \theta, \quad 0<\theta<\frac{1}{2} \pi,
$$

then $\theta$ should be bounded away from 0 and $\frac{1}{2} \pi$.

\section{ACKNOWLEDGMENT}

The author would like to thank the referees for their comments on the first version of the paper.

\section{BIBLIOGRAPHY}

1. M. Abramowitz and I. A. Stegun, Handbook of mathematical functions with formulas, graphs and mathematical tables, Nat. Bur. Standards Appl. Math. Series, no. 55, U.S. Government Printing Office, Washington, D.C., 1964.

2. D. J. Best and D. E. Roberts, Algorithm AS 91: The percentage points of the $\chi^{2}$ distribution, Appl. Statist. 24 (1975), 385-388.

3. J. M. Blair, C. A. Edwards, and J. H. Johnson, Rational Chebyshev approximations for the inverse of the error function, Math. Comp. 30 (1976), 827-830.

4. T. A. Burgin, The gamma distribution and inventory control, Oper. Res. Q. 26 (1975), $507-$ 525.

5. A. R. DiDonato and A. H. Morris, Jr., Computation of the incomplete gamma functions, ACM Trans. Math. Software 12 (1986), 377-393.

6. H. E. Fettis, An asymptotic expansion for the upper percentage points of the $\chi^{2}$ distribution, Math. Comp. 33 (1979), 1059-1064.

7. A. J. Strecok, On the calculation of the inverse of the error function, Math. Comp. 22 (1968), 144-158.

8. N. M. Temme, The asymptotic expansions of the incomplete gamma functions, SIAM J. Math. Anal. 10 (1979), 757-766.

9. __ The uniform asymptotic expansion of a class of integrals related to cumulative distribution functions, SIAM J. Math. Anal. 13 (1982), 239-253.

CWI, KruislaAn 413, 1098 SJ Amsterdam, The Netherlands

E-mail address: nicot@cwi.nl 